INPLASY

PROTOCOL

To cite: Zhou et al. Acupoint injection therapy for diabetic retinopathy: a protocol for systematic review and metaanalysis. Inplasy protocol 2020120045. doi:

10.37766/inplasy2020.12.0045

Received: 08 December 2020

Published: 08 December 2020

Corresponding author:

Yanni Zhou

403021606@qq.com

Author Affiliation:

Chengdu University of TCM

Support: The NSFC (No.

81373644).

Review Stage at time of this submission: Preliminary

searches.

Conflicts of interest:

None.

\section{Acupoint injection therapy for diabetic retinopathy: a protocol for systematic review and meta-analysis}

Zhou, YN1; LI, H²; Luo, LS33; Chen, Y4; Chen, Q5; Bian, W66.

Review question / Objective: The purpose of this study is to evaluate the efficacy of acupoint injection for diabetic retinopathy.

Condition being studied: Diabetes retinopathy (DR) is one of the most common microvascular complication of diabetes. Without further treatment, diabetic retinopathy can lead to neovascularization, vitreous hemorrhage or anterior retinal hemorrhage, which will lead to severe vision loss and increase the risk of blindness. Its high prevalence and associated high risk of vision loss lead it to the major global health burden. Therefore, early prevention and treatment are necessary. However, conventional treatment options are limited and mainly include glucose control, blood pressure and lipid control, aspirin, and lifestyle modifications. No approaches have been developed specifically to prevent and treat DR.Recent clinical and experimental studies have shown that acupoint injection is effective in the prevention and treatment of DR. Acupoint injection therapy is a kind of traditional Chinese medicine technology, which injects Chinese and Western medicine into corresponding acupoints of human body to achieve the purpose of treating diseases.Through this therapy, acupoint penetration, drug efficacy and acupuncture stimulation can be more effective, and the clinical symptoms of DR can be significantly improved. Thus, we intend to conduct a systematic review and meta-analysis to testify acupoint injection therapy for diabetic retinopathy.

INPLASY registration number: This protocol was registered with the International Platform of Registered Systematic Review and Meta-Analysis Protocols (INPLASY) on 08 December 2020 and was last updated on 08 December 2020 (registration number INPLASY2020120045).

\section{INTRODUCTION}

Review question / Objective: The purpose of this study is to evaluate the efficacy of acupoint injection for diabetic retinopathy.
Rationale: A lot of clinical studies have reported that acupoint injection has a good effect on the treatment of diabetic retinopathy, but there is no relevant systematic review or meta-analysis. 
Condition being studied: Diabetes retinopathy (DR) is one of the most common microvascular complication of diabetes. Without further treatment, diabetic retinopathy can lead to neovascularization, vitreous hemorrhage or anterior retinal hemorrhage, which will lead to severe vision loss and increase the risk of blindness. Its high prevalence and associated high risk of vision loss lead it to the major global health burden. Therefore, early prevention and treatment are necessary. However, conventional treatment options are limited and mainly include glucose control, blood pressure and lipid control, aspirin, and lifestyle modifications. No approaches have been developed specifically to prevent and treat DR.Recent clinical and experimental studies have shown that acupoint injection is effective in the prevention and treatment of DR. Acupoint injection therapy is a kind of traditional Chinese medicine technology, which injects Chinese and Western medicine into corresponding acupoints of human body to achieve the purpose of treating diseases.Through this therapy, acupoint penetration, drug efficacy and acupuncture stimulation can be more effective, and the clinical symptoms of DR can be significantly improved. Thus, we intend to conduct a systematic review and meta-analysis to testify acupoint injection therapy for diabetic retinopathy.

\section{METHODS}

Participant or population: We will include patients with a diagnosis of diabetic retinopathy. The diagnostic criteria of diabetic retinopathy in the selected literature should meet the internationally recognized standards.

Intervention: Interventions included treatment with acupoint injection. The drug types for acupoint injection, the acupuncture points for injection, treatment frequency, and duration of treatment will not be restricted. The use of acupoint injection is the only difference between intervention and control.
Comparator: The control interventions will include: positive interventions, placebo, no intervention. The choice of specific forms are as follows: (1). acupoint injection VS positive interventions; (2). acupoint injection + positive interventions VS positive interventions; (3). acupoint injection VS placebo; (4). acupoint injection VS no intervention.

Study designs to be included: All relevant randomised controlled trials (RCTs) and quasi-randomized controlled trials (q-RCTs) will be included.

Eligibility criteria: Inclusion criteria -Patients should be diagnosed as DR according to the currently recognized diagnostic criteria. There will be no restrictions on the length of treatment and duration of follow-up. The language of the trials to be included only Chinese or English. Without any date of dissemination or restriction of publication type. Exclusion criteria- Following studies will be excluded:1. Repeated publications;2. Review of literature and cases; 3 . Incomplete literature; 4. Non-randomized controlled trials; 5. Patients combined with other basic diseases.

Information sources: We will electronically search the randomized controlled trials in the following databases: including the PubMed, Embase, the Cochrane Library, China National Knowledge Infrastructure (CNKI), Web of Science, Chinese Scientific Journal Database(VIP), Wanfang Database and Chinese Biomedical Literatures Database (CBM) with a language limitation of English and Chinese until November 2020. The search strategy that will be run in the PubMed and adjusted to fit the other database when necessary.

Main outcome(s): The primary outcomes were the effective rate. The effective rate was defined as the number of patients who showed improvement in retinal vascularrelated abnormalities.

Additional outcome(s): 1.visual acuity ; 2 . retinal vascular abnormalities: 
hemorrhage areas, exudates, capillary nonperfusion areas, hemorheological indicators; 3. mean defect of visual field;

4. glycated haemoglobin; 5. adverse events.

Quality assessment / Risk of bias analysis: Two independent reviewers will use the Cochrane "bias risk assessment" tool to assess the bias risk of the selected trials. The tool assesses the risk of bias mainly in the following 7 aspects: random sequence generation, allocation concealment, the blinding method for patients, researchers and outcomes assessors, incomplete result data, and selective reports. As recommended by the Cochrane manual, the risk of bias in each of these areas will be assessed as low or high depending on whether the criteria were met or not met, and the lack of information will be recorded as unclear. Differences will be resolved by discussion with the third author until consensus be achieved.

Strategy of data synthesis: We will use RevMan5.3 software for meta-analysis. For dichotomous data (e.g., effective and ineffective), we will calculate risk ratio (RR) and $95 \%$ confidence intervals (Cls). For continuous data, when the measurement method and unit are consistent, we will calculate the weighted mean difference (WMD) and $95 \% \mathrm{CIs.} \mathrm{When} \mathrm{the}$ measurement methods and units are inconsistent or the mean values of different experiments differ greatly, we will use the standardized mean difference (SMD) and $95 \%$ Cls as the composite statistics. I2 value and $P$-value will be used to test the degree of heterogeneity. When $\mathrm{P}>0.1, \mathrm{I}^{2}<$ $50 \%$, no heterogeneity was considered between the studies and the fixed effect model will be used for statistical analysis; otherwise, the random effect model will be used. If there was significant clinical heterogeneity between studies, only descriptive analysis was performed.For a study with incomplete result data, we will try to contact the first author.

Subgroup analysis: Consideration of the possible impact of cupping types, intervention methods, and outcome measurements to the conspicuous heterogeneity, subgroup analysis will be conducted.

Sensibility analysis: The robustness and reliability of the review outcome will be judged by sensitivity analysis in the statistical model, sample size, missing data, and others.

Country(ies) involved: China.

Keywords: acupoint injection; diabetic retinopathy; efficacy; protocol.

\section{Contributions of each author:}

Author 1 - Yanni Zhou - extract data and drafted the manuscript.

Author 2 - Hui Li - contributed to the development of the selection criteria, and the risk of bias assessment strategy.

Author 3 - Lisi Luo - contributed to the development of the selection criteria, and the risk of bias assessment strategy.

Author 4 - Yue Chen - provided statistical expertise.

Author 5 - Qiang Chen - use the software to analyze the data.

Author 6 - Wei Bian - read, provided feedback and approved the final manuscript. 